\title{
Correction: Extracellular vesicles derived from miR-199a-5p-modified adipose-derived mesenchymal stem cells alleviate immune thrombocytopenia by inhibiting $\mathrm{T}$ helper 17 differentiation
}

Jianqin Li $\mathbb{D} \cdot$ Yalin Xia $\cdot$ Xiaoru Fan $\cdot$ Xiaofang Wu Feiyun Yang $\cdot$ Shaoyan Hu $\cdot$ Zhaoyue Wang

Published online: 18 May 2021

(C) The Author(s), under exclusive licence to United States and Canadian Academy of Pathology 2021

Correction to: Laboratory Investigation

https://doi.org/10.1038/s41374-020-00515-z
The original version of this article unfortunately containd a mistake in an author name. Yanlin Xia should be Yalin Xia. The originial article has been corrected. 